\title{
Adhesion Awareness: A National Survey of Surgeons
}

\author{
Marc H. F. Schreinemacher - Richard P. ten Broek • \\ Erica A. Bakkum - Harry van Goor • \\ Nicole D. Bouvy
}

Published online: 3 September 2010

(C) The Author(s) 2010. This article is published with open access at Springerlink.com

\begin{abstract}
Background Postoperative adhesions are the most frequent complication of abdominal surgery, leading to high morbidity, mortality, and costs. However, the problem seems to be neglected by surgeons for largely unknown reasons.

Methods A survey assessing knowledge and personal opinion about the extent and impact of adhesions was sent to all Dutch surgeons and surgical trainees. The informedconsent process and application of antiadhesive agents were questioned in addition.

Results The response rate was $34.4 \%$. Two thirds of all respondents $(67.7 \%)$ agreed that adhesions exert a clinically relevant, negative effect. A negative perception of adhesions correlated with a positive attitude regarding adhesion prevention $(\rho=0.182, p<0.001)$. However, underestimation of the extent and impact of adhesions resulted in low knowledge scores (mean test score 37.6\%). Lower scores correlated with more uncertainty about indications for antiadhesive agents which, in turn, correlated with never having used any of these agents $(\rho=0.140, p=0.002$; $\rho=0.095, p=0.035$; respectively). Four in 10 respondents $(40.9 \%)$ indicated that they never inform patients on adhesions and only $9.8 \%$ informed patients routinely.
\end{abstract}

M. H. F. Schreinemacher · N. D. Bouvy $(\bowtie)$

Department of General Surgery, Maastricht University Medical Centre, P.O. Box 5800, 6202 AZ Maastricht, The Netherlands

e-mail: n.bouvy@mumc.nl

R. P. ten Broek - H. van Goor

Department of General Surgery, Radboud University Nijmegen Medical Centre, Nijmegen, The Netherlands

E. A. Bakkum

Department of Obstetrics and Gynecology, Onze Lieve Vrouwe Gasthuis, Amsterdam, The Netherlands
A majority of surgeons (55.9\%) used antiadhesive agents in the past, but only a minority $(13.4 \%)$ did in the previous year. Of trainees, $82.1 \%$ foresaw an increase in the use of antiadhesive agents compared to $64.5 \%$ of surgeons $(p<0.001)$. Conclusions The magnitude of the problem of postoperative adhesions is underestimated and informed consent is provided inadequately by Dutch surgeons. Exerting adhesion prevention is related to the perception of and knowledge about adhesions.

\section{Introduction}

Postoperative adhesions occur in about $90 \%$ of all patients undergoing abdominal surgery and lead to at least one readmission for a third of these patients in the following 10 years $[1,2]$. Adhesions become clinically apparent in the form of chronic abdominal pain, female infertility, and small-bowel obstruction [3, 4]. Furthermore, adhesions can seriously complicate subsequent surgery $[5,6]$. Therefore, postoperative adhesions should not merely be regarded as a side effect of abdominal surgery but as the most common complication caused by abdominal surgery.

Regardless of an open or a laparoscopic approach, the surgical treatment of adhesions induces the reformation as well as new formation of adhesions [7, 8]. Hence, adhesion prevention is of key importance. For obvious reasons, reducing surgical trauma by meticulous surgical technique is the primary step that needs to be exerted at all times. However, performing surgery implies surgical trauma to some extent but it can be further reduced by other means, e.g., using powder-free gloves, wetting tissues, and reducing operative time [9]. The use of adhesion barriers seems inevitable to obtain further adhesion prevention. A local barrier composed of hyaluronic acid and carboxymethylcellulose (Seprafilm ${ }^{\circledR}$, 
Genzyme, Cambridge, MA, USA) has proven effective in reducing adhesions in various open general surgery studies [10]. Previous reviews have also shown significant benefit with the use of Interceed ${ }^{\circledR}$ membrane (Ethicon 360, Johnson \& Johnson, New Brunswick, NJ, USA), composed of oxidized regenerated cellulose, in open gynecologic surgery [11]. Administration of an icodextrin solution $\left(\right.$ Adept $^{\circledR}$, Baxter Healthcare Corp., Deerfield, IL, USA) that spreads throughout the peritoneal cavity has shown adhesion reductive capacity in benign laparoscopic gynecologic surgery [12].

In contrast to most surgical complications, the risk of adhesion-related morbidity remains for many years and complications are often not followed up by the primary surgeon. In addition, symptoms of adhesion-related complications vary and a fully effective remedy has not yet been discovered. All these factors have probably resulted in an undervaluation of postoperative adhesions by surgeons. This in turn explains why adhesions are mentioned only sporadically during the informed-consent process $[13,14]$. Nevertheless, failure to do so can be regarded as an omission of the doctor's duty of care and has already resulted in successful negligence claims [15].

In spite of the extent and impact of postoperative adhesions, we are under the impression that surgeons lack sufficient awareness about this most common complication. Moreover, they seem to provide inadequate informed consent on and take insufficient preventive actions against adhesions. However, until now, no data has been available to substantiate these assumptions. Therefore, we conducted a nationwide survey assessing the awareness of and behavior toward adhesions among Dutch surgeons and surgical trainees.

\section{Materials and methods}

Design of the survey

A steering group of 11 general and gynecologic surgeons with a special interest in adhesions and its associated morbidity (Dutch Adhesion Group) conceived a first set of survey questions. These questions were edited by two independent researchers, both experts in survey and multiple-choice test construction. Subsequently, five surgeons and three surgical trainees tested the survey for indistinctness and leading questions. After making adjustments, the survey was reviewed again and consecutively approved by the steering group, the independent researchers, and the test group of surgeons and trainees. The survey consisted of 55 multiple-choice questions, four open-ended questions, and four optional questions with a total word count of 716 . In its final layout, both an electronic online version (six web pages) and a printed version (three pages) were available in Dutch (see the Appendix for a translated version).

\section{Knowledge test}

Eight multiple-choice questions concerning the prevalence and morbidity of adhesions were formulated based on up-to-date and best-available evidence. The following statements were considered correct:

- Approximately $70 \%$ of small-bowel obstructions are due to postoperative adhesions [16-18].

- The 5-year readmission rate after operative procedures of the colon or rectum directly related to postoperative adhesions is approximately 5\% [19-21].

- The 10-year readmission rate after any abdominal surgery probably or directly related to postoperative adhesions is approximately $30 \%[1,2]$.

- Inadvertent enterotomy during adhesiolysis occurs in $20 \%$ of patients with a history of abdominal surgery $[5,6]$.

- A total colonic resection has the highest risk of adhesion-related morbidity compared with a partial small-bowel resection, an appendectomy, or a resection of the rectum $[2,21]$.

- Age above 60 years is associated with fewer adhesions, a history of abdominal surgery with more adhesions, and a history of Crohn's disease with no difference in adhesion formation $[2,21]$.

\section{Survey distribution}

We aimed to distribute the survey among all officially registered Dutch surgeons and trainees $(n=1,282$ and 432 , respectively). Contact details were retrieved from the 2008 annual report of the Dutch Association for Surgery from the section of regular members $(n=1,009)$ and members of the association of surgical trainees $(n=446)$. We approached the surgeons and trainees by electronic mail (e-mail) or by postal mail when no or no valid e-mail address was available. A personalized mail was sent on Tuesday (at 6:00 a.m. in case of e-mail) and a reminder sent the next Tuesday (at 7:00 p.m. in case of e-mail) when no response was recorded yet. As an incentive, five portable audio players and 40 pens with inbuilt laser pointer and USB stick were raffled among all respondents. The survey closed 3 weeks after the first mailing.

Data analysis

Only surveys more than $80 \%$ complete, excluding optional questions, were included. We defined subgroups of respondents as trainees, general surgeons, gastrointestinal 
surgeons, or other surgeons. Proportions were compared using $\chi^{2}$ tests. Comparisons between groups were performed using the Mann-Whitney $U$ test, the Wilcoxon signed-rank test, or the Kruskal-Wallis test with post-hoc Bonferroni correction. Knowledge test scores were compared with Student's $t$ tests and ANOVA with post-hoc Bonferroni correction. Correlations were calculated using Spearman's rank correlation and a $p<0.050$ was considered significant. Statistics were performed using SPSS $^{\circledR}$ version 15.0 (SPSS, Inc., Chicago, IL, USA).

\section{Results}

A total of 1,455 surgeons and trainees were contacted by email $(83.8 \%)$ or postal mail $(16.2 \%)$. Twenty-three physicians indicated not to participate. After 1 week, 352 surveys were collected, and there were 523 surveys at close of the survey. Of these, 22 incomplete surveys (4.2\%) were rejected, resulting in a response rate of $34.4 \%$ (501 surveys, $98 \%$ complete) representing $90.7 \%$ of all Dutch surgical departments $(98.3 \%$ of all teaching and $81.3 \%$ of all nonteaching departments). Response rates were comparable for e-mail and postal mail (35.3 vs. 29.8\%, respectively, $p=0.102$ ), but higher for surgeons than for trainees (Table 1). The survey was completed by a comparable number of trainees $(n=131)$, general surgeons $(n=130)$, gastrointestinal surgeons $(n=116)$, and other surgeons $(n=124)(p=0.767)$.

Opinion on adhesions

About two thirds $(67.7 \%)$ of all respondents agreed that adhesions exert a clinically relevant and predominantly negative effect. The proportion was significantly higher for trainees than for surgeons (75.6 vs. $64.9 \%$, respectively,

Table 1 Respondents $(n=501)$

\begin{tabular}{llll}
\hline & Trainees & Surgeons & $p$ Value \\
\hline $\begin{array}{l}\text { Response rate } \\
(\%)(n)\end{array}$ & $29.4 \%(131$ of 446$)$ & $36.7 \%(370$ of 1,009$)$ & 0.007 \\
$\begin{array}{l}\text { Experience, } \\
\text { mean (SD) } \\
\text { (years) }\end{array}$ & $4.3(1.6)$ & $13.2(9.6)$ & n.a. \\
$\begin{array}{l}\text { Full-time } \\
\text { employment } \\
(\%)(n)\end{array}$ & $89.9 \%(116)$ & $88.8 \%(316)$ & 0.718 \\
$\begin{array}{l}\text { Academic } \\
\text { hospital } \\
\text { employment } \\
(\%)(n)\end{array}$ & $33.6 \%(44)$ & $25.8 \%(95)$ & 0.088 \\
\end{tabular}

$S D$ standard deviation; n.a. not applicable

All $\chi^{2}$ tests $p=0.025)$. A small group $(6.0 \%)$ indicated that adhesions exert a clinically relevant and predominantly positive effect. Half of all respondents $(50.6 \%)$ considered adhesiolysis for treating pain not effective, whereas $26.2 \%$ considered it effective.

Awareness of adhesions and their associated morbidity

Respondents scored a mean of $37.6 \%$ correct answers on the knowledge test, with trainees scoring slightly but significantly higher than general surgeons (39.9 vs. $34.6 \%$, $p=0.032$ ). Only $6.9 \%$ of respondents reported a correct 10 -year readmission rate after abdominal surgery probably or directly related to adhesions (30\%), whereas $69.0 \%$ thought it was $10 \%$ or lower. Also, $62.9 \%$ underestimated the percentage of small-bowel obstructions caused by adhesions, reporting a percentage of $50 \%$ or lower. On the other hand, a vast majority of respondents (87.6\%) correctly indicated that a history of abdominal operations is associated with increased adhesion-related morbidity. The knowledge test score did not correlate with respondents' opinion on adhesions $(\rho=0.010, p=0.830)$.

\section{Informed consent}

One in 10 respondents $(9.8 \%)$ reported that they routinely include adhesions or related morbidity in the informed consent information for both laparotomies and laparoscopies. Yet, $40.9 \%$ of all respondents reported that they never mention it at all (Fig. 1). No correlation was observed with opinion on adhesions or knowledge test score $(\rho=0.031$, $p=0.497 ; \rho=0.016, p=0.730$; respectively). Trainees and nonacademic surgeons provided adhesion information during informed consent less often than surgeons and academic surgeons, respectively (both $p=0.002$ ). Furthermore, providing information on adhesions before a

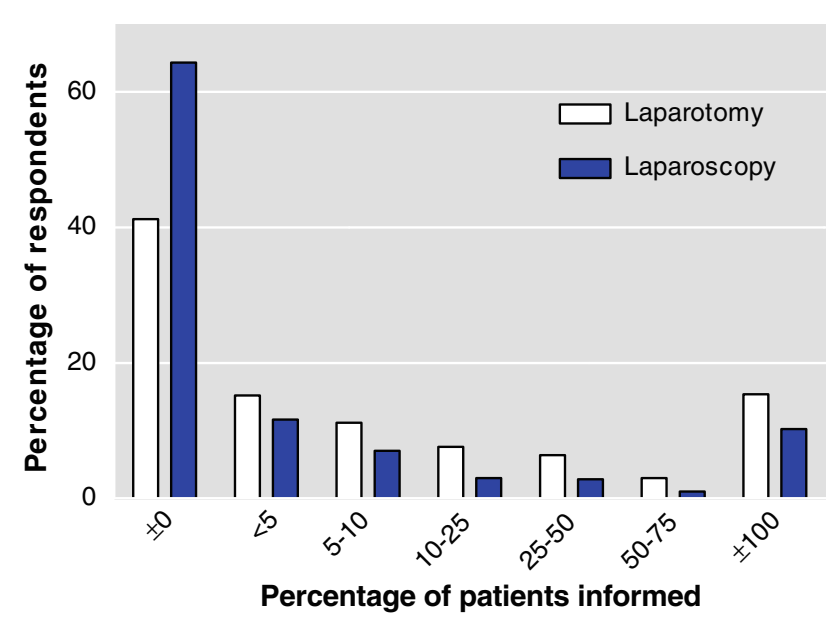

Fig. 1 Informed consent 
laparoscopic procedure was done less often in comparison with laparotomy $(p<0.001)$. Informing fewer patients before a laparoscopy correlated with a greater belief that laparoscopy is a means to limit adhesion formation $(\rho=0.186, p<0.001)$.

\section{Adhesion prevention}

Four in 10 respondents (39.1\%) expressed a positive opinion on adhesion prevention, $22.4 \%$ expressed a negative one. In addition, a positive opinion correlated with a negative view of adhesions $(\rho=0.182, p<0.001)$. All respondents, except gastrointestinal surgeons, believed more strongly in adhesion prevention for specific indications than for all abdominal surgery $(p<0.001)$. Significantly more surgeons than trainees believed that a meticulous surgical technique minimizes adhesions ( 83.5 vs. $65.6 \%, p<0.001$ ). Similarly, significantly more gastrointestinal than nongastrointestinal surgeons believed that laparoscopy reduces adhesion formation (90.5 vs. $72.0 \%, p<0.001)$ (Fig. 2).

\section{Antiadhesive agents}

Of all respondents, $26.5 \%$ expressed a positive attitude toward antiadhesive agents and $29.1 \%$ expressed a negative one (Fig. 3). Although a majority of surgeons (55.9\%) had used at least one of these agents, only a minority did in the previous year (13.4\%). Significantly higher proportions of gastrointestinal and academic surgeons used an antiadhesive agent in the previous year compared to general and nonacademic surgeons, respectively ( 23.0 vs. $9.0 \%, p=0.001$; 23.9 vs. $10.5 \%, p<0.0001$; respectively). Not using antiadhesive agents any longer showed no significant associations with knowledge test score, opinion on adhesions, adhesion prevention, or antiadhesive agents $(p=0.622$, $p=0.431, p=0.283, p=0.209$, respectively). Most surgeons used Adept $^{\circledR}$ (8.9\% ever, $8.5 \%$ last year) and Seprafilm ${ }^{\circledR}$ (33.7\% ever, $5.4 \%$ last year). In the group of surgeons who used antiadhesive agents, $78.8 \%$ did so in adhesionrelated laparotomies, $29.2 \%$ in abdominal wall surgery, and $21.8 \%$ in (sub)total colectomies. In general, use of products for high-risk operations regarding adhesion formation was higher than for low-risk operations $(p<0.001)$. Uncertainty about when to use these products correlated with never having used any products $(\rho=0.095, p=0.035)$ and lower knowledge test scores $(\rho=0.140, p=0.002)$.

Of all trainees, $82.1 \%$ predicted that the use of antiadhesive agents will increase compared with $64.5 \%$ of all surgeons $(p<0.001)$. Such opinion correlated with a negative view of adhesions ( $\rho=0.141, p=0.002)$ and with a more positive view in terms of cost-benefits $(\rho=0.148, p=$ 0.001). Most respondents anticipated that new antiadhesive agents would come to the market and that the evidence either for or against adhesion prevention would increase.

\section{Discussion}

Adhesions and related complications lead to substantial morbidity and mortality, with increased medical costs [22, 23]. This nationwide survey shows that two of three Dutch surgeons recognize adhesions as a clinically relevant and negative entity. However, readmission rates and smallbowel obstructions caused by adhesions are heavily underestimated. Moreover, the informed-consent process and application of antiadhesive agents are not in line with the extent and impact of postoperative adhesions. Therefore, it can be concluded that the knowledge and awareness of and the behavior toward adhesions is limited among Dutch surgeons and surgical trainees.
Fig. 2 Techniques to minimize adhesions

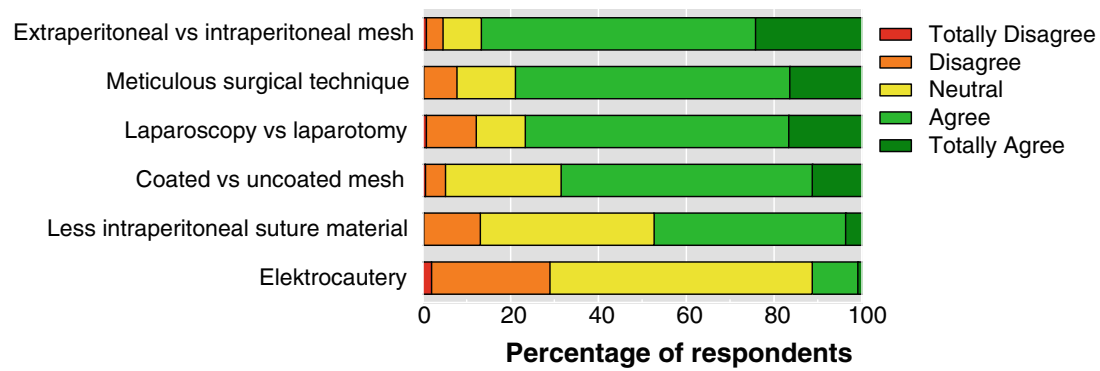

Fig. 3 Opinion on antiadhesive agents

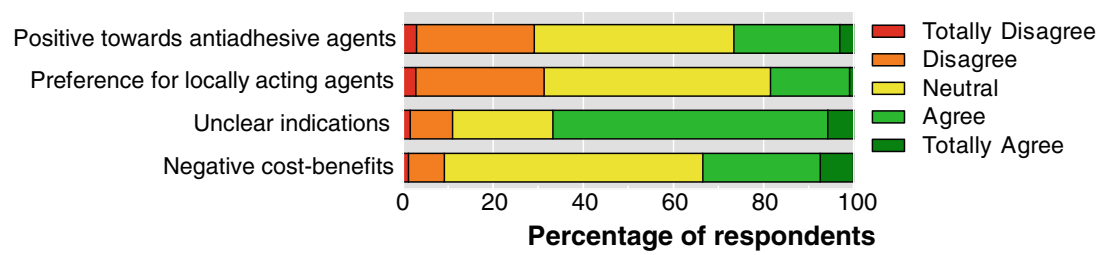


At least $40 \%$ of respondents did not inform any patients about postoperative adhesions or related morbidity and only very few informed patients routinely. This corresponds with the recent finding that more than $90 \%$ of consent forms lack this information [13]. In contrast, the risks for hemorrhage and infection after abdominal surgery are almost invariably discussed during the informed-consent process. Adhesion-related complications share nearly all features of these complications, including the risk of death, but may occur many years after the operation. Thus, it is essential to discuss adhesions as a possible complication during the informed-consent process. In addition, in case of any reoperation, a high risk of inadvertent organ damage exists and should be discussed prior to surgery as well. These recommendations apply also for laparoscopic procedures since laparoscopy has not been proven to reduce adhesion-related morbidity compared with laparotomy, though definite studies are lacking [24].

Current surgical trainees consider adhesions a negative drawback of surgery more often, have a slightly better understanding of the extent of the problem, rely less on surgical technique to reduce adhesions, and have a higher belief in an increasing use of antiadhesive agents, compared to surgeons. In contrast, they seem to inform fewer patients about adhesions or adhesion-related morbidity compared to current surgeons. The reason for this is unclear, but this behavior may change when they have more responsibilities and follow-up their own patients more closely. The finding is of interest for the future and might mandate (educational) interventions with this group of young surgeons before they face legal claims. The significantly lower response rate of surgical trainees compared to surgeons can be explained by the higher number of trainees' contact details than the number of officially registered trainees for general surgery. This is probably due to the fact that plastic and orthopedic surgery trainees share the first 2 years of training.

The current study is the first to evaluate awareness and behavior of surgeons regarding adhesions. Recently, two similar studies reported on adhesion awareness among gynecologic surgeons in the United Kingdom and Germany $[14,25]$. Little over half of those respondents agreed that adhesions are the most common complication after abdominal surgery. This is in line with our finding that the impact of adhesions is underestimated. Yet, around three in four gynecologic surgeons indicated that they inform patients routinely about adhesions and about half of the respondents stated that they use antiadhesive agents regularly. This clearly surpasses the current behavior of Dutch surgeons and reflects a higher awareness of adhesions among gynecologic surgeons. Nevertheless, the $8.8 \%$ response rate of the British gynecologists and the $33.5 \%$ response rate of German gynecologic departments might reflect a selection bias.
Approximately $80 \%$ of respondents agreed that an extraperitoneal mesh, meticulous surgical technique, and laparoscopy reduce adhesions. Only a quarter of surgeons expressed a positive opinion toward antiadhesive agents. In addition, only one in 10 surgeons used an agent in the past year, with Adept $\left.^{(}\right)$taking a small lead over Seprafilm ${ }^{\circledR}$. Adept ${ }^{\circledR}$ is a liquid acting throughout the whole abdomen; Seprafilm $^{\circledR}$ is a site-specific barrier film. The efficacy of Seprafilm ${ }^{\circledR}$ has already been evidenced in general surgery, whereas for Adept ${ }^{\circledR}$ efficacy has been shown in laparoscopic gynecologic surgery and results in general surgery are awaited [10, 12]. Both agents have been shown to be safe in general abdominal surgery, though application of Seprafilm ${ }^{\circledR}$ on bowel anastomoses should be avoided [10, 26, 27]. Although current agents do not provide complete prevention of postoperative adhesions, it is worth considering their use in high-risk surgery such as colorectal procedures. Even a relative decrease in adhesions might entail benefits for the patient and the surgeon. Interestingly, uncertainty about indications for antiadhesive agents was correlated with never having used any agents and with lower knowledge scores.

This study demonstrates that a change in behavior is needed among surgeons and trainees. Patients have to be informed of the risks of adhesions routinely and the application of clinically available antiadhesive agents should at least be considered in specific abdominal surgery [28]. However, to obtain changes in behavior, both knowledge and attitudes have to be addressed [29]. Barriers for improving knowledge include accessibility and volume of information; attitudes can be affected by a lack of agreement or poor methodology in evidence, but also by personal beliefs and experiences. Therefore, attempts to obtain sustainable changes in behavior have to target multiple aspects. Moreover, breakthrough results from basic research should be translated to clinically applicable agents [30].

Some limitations of this study should be noted. First of all, the $34.4 \%$ response rate might reflect a selection bias, possibly providing more positive results. Furthermore, no elaborate nonresponse analysis could be performed due to the lack of any central database comprising detailed information on Dutch surgeons. On the other hand, responses were recorded from $90.7 \%$ of all Dutch surgical departments. In addition, response rates were markedly higher than in the recent survey among gynecologic surgeons in the UK and a survey in the USA on work, stress, and research among academic surgeons (8.8 and 22.7\%, respectively) [14, 31]. Our relatively high response rate might be due to the raffle, which is known to increase response rates without affecting response quality [32, 33].

The knowledge test was based on up-to-date and bestavailable evidence selected by the steering group of general and gynecologic surgeons. Several rounds of pilot testing were conducted to ensure comprehensibility and face and 
content validity of the survey. However, although most of the knowledge questions were based on very large cohort studies, some still consider the consistently found high morbidity controversial. Yet, even if the true morbidity would be lower, many of the respondents would still underestimate the magnitude of the problem.

Finally, this study surveyed only Dutch surgeons and surgical trainees, but results are likely to be generalizable to surgeons worldwide; adhesions are encountered after all abdominal surgeries and the associated morbidity, mortality, and costs are comparably high in different countries $[23,34,35]$.

As for the future, action must be taken to improve knowledge, attitude, and behavior concerning adhesions among Dutch surgeons and surgical trainees. Specialty courses could be developed and rewarded credits; compulsory classes on adhesions and antiadhesive agents should be embedded in the surgical training program. Nonetheless, surgeons represent only one of the many parties involved in adhesion awareness. Attention must also be given to the other specialists operating in the abdominal cavity, patients, hospitals, antiadhesive agents manufacturers, and health insurance companies in order to improve awareness of and behavior toward adhesions. In order to follow-up on the actions, the results of this survey can serve as a benchmark for later research.

Acknowledgments The Dutch Adhesion Group consists of Mrs. E. A. Bakkum (gynecologic surgeon, Amsterdam), Mrs. N. D. Bouvy (surgeon, Maastricht), F. P. Dijkhuizen (gynecologic surgeon, Arnhem), H. van Goor (surgeon, Nijmegen), F. W. Jansen (gynecologic surgeon, Leiden), J. Jeekel (surgeon, Rotterdam), R. F. Kruitwagen (gynecologic surgeon, Maastricht), M. M. Reijnen (surgeon, Arnhem), E. J. van Santbrink (gynecologic surgeon, Rotterdam), D. J. Swank (surgeon, Gouda), and Mrs. G. C. Trimbos-Kemper (gynecologic surgeon, Leiden).

Disclosures Portable audio players and pens with built-in laser pointer and USB stick were supplied unconditionally and free from any imprints by Johnson \& Johnson Medical, Amersfoort, The Netherlands. None of the authors holds any financial interest in Johnson \& Johnson Medical nor in any of the companies whose products are mentioned in this study.

Open Access This article is distributed under the terms of the Creative Commons Attribution Noncommercial License which permits any noncommercial use, distribution, and reproduction in any medium, provided the original author(s) and source are credited.

\section{Appendix: Adhesions survey}

Personal data

- You are a (multiple responses possible): trainee, general surgeon, oncologic surgeon, vascular surgeon, gastrointestinal surgeon, pediatric surgeon, trauma surgeon, other...

- How many years of work experience (as a trainee or as a surgeon) do you have?...

- Do you currently work in an academic or nonacademic hospital? Yes/No

- Do you currently work full-time or part-time? Yes/No

Prevalence and morbidity of adhesions

- Adhesions are not of clinical interest. Totally disagree/ Disagree/Neutral/Agree/Totally agree

- Adhesions have more beneficial than detrimental effects. Totally disagree/Disagree/Neutral/Agree/Totally agree

- Adhesiolysis for complaints of pain is not effective. Totally disagree/Disagree/Neutral/Agree/Totally agree

- A proportion of all small-bowel obstructions is caused by postoperative adhesions. This proportion is closest to: $30 \% / 50 \% / 70 \% / 90 \%$

- Within 5 years after an operation on the colon or rectum a certain percentage of patients will be readmitted because of morbidity directly related to adhesions. This percentage is closest to: $2 \% / 5 \% / 10 \% / 20 \%$

- Within 10 years after an abdominal operation a certain percentage of patients will be readmitted because of morbidity possibly related to adhesions. This percentage is closest to: $5 \% / 10 \% / 20 \% / 30 \%$

- Inadvertent bowel lesions caused by adhesiolysis occur in a certain percentage of patients with a history of an abdominal operation. This percentage is closest to: $5 \% /$ $10 \% / 20 \% / 30 \%$

- Which of the following procedures carries with it the highest risk for adhesion-related morbidity? Partial smallbowel resection/appendectomy/total (procto)colectomy/ proctectomy

- Some patient characteristics have been proven to influence adhesion formation. What do you think is the influence of:

- Age above 60 years: less adhesions/no effect/more adhesions

- History of abdominal operations: less adhesions/no effect/more adhesions

- Crohn's disease: less adhesions/no effect/more adhesions

Informed consent

- How many patients do you inform about adhesions or adhesion-related morbidity as a possible complication after 
- laparotomy? virtually none/<5\%/5-10\%/10-25\%/

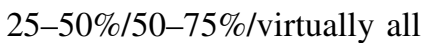

- laparoscopy? virtually none/<5\%/5-10\%/10-25\%/

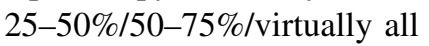

Opinion on adhesion prevention

- You do not believe in adhesion prevention. Totally disagree/Disagree/Neutral/Agree/Totally agree

- You would like to apply adhesion prevention in all abdominal operations. Totally disagree/Disagree/Neutral/Agree/Totally agree

- You would like to apply adhesion prevention only in certain indications. Totally disagree/Disagree/Neutral/ Agree/Totally agree

- Laparoscopic surgery causes fewer adhesions than open surgery. Totally disagree/Disagree/Neutral/Agree/Totally agree

- Meticulous surgical technique (tissue handling, avoiding gauzes) reduces adhesions. Totally disagree/Disagree/Neutral/Agree/Totally agree

- Extraperitoneal mesh position causes fewer adhesions than the intraperitoneal mesh position. Totally disagree/ Disagree/Neutral/Agree/Totally agree

- A coated mesh causes fewer adhesions than an uncoated mesh. Totally disagree/Disagree/Neutral/Agree/Totally agree

- Electrocautery causes fewer adhesions. Totally disagree/Disagree/Neutral/Agree/Totally agree

- Less intraperitoneal suture material reduces adhesions. Totally disagree/Disagree/Neutral/Agree/Totally agree

Opinion on antiadhesive agents

- You don't believe in antiadhesive agents. Totally disagree/Disagree/Neutral/Agree/Totally agree

- You experience a lack of clarity about when to use an antiadhesive agent. Totally disagree/Disagree/Neutral/ Agree/Totally agree

- You prefer using a locally acting antiadhesive agent over an agent that acts throughout the whole abdomen. Totally disagree/Disagree/Neutral/Agree/Totally agree

- You think the costs do not outweigh the possible benefits of antiadhesive agents. Totally disagree/Disagree/ Neutral/Agree/Totally agree

- Which factors influence your belief in adhesion prevention?...

\section{Using antiadhesive agents}

- Have you ever used:

Interceed, Seprafilm, Adept, Spraygel, Hyalobarrier, Prevadh, Ringers lactate (not regarded an antiadhesive agent),
Intercoat, Other... For all agents: Never used, but would like to/Never used/Used in the past year/Ever used

- With which operation have you ever used antiadhesive agents? Never/Seldom/Regularly/Often/Very often

Relaparotomy because of adhesion-related morbidity, Pancreatic or duodenal surgery, Partial small-bowel resection, Ileostomy creation, Appendectomy, Partial colectomy, Panproctocolectomy, Laparoscopic colon surgery, Rectal operation, Abdominal wall surgery, Aortic bifurcation prosthesis, Other...

- How many times are antiadhesive agents used by you and your colleagues in your hospital?

- \% of all laparotomies...

- \% of all laparoscopies...

- Do you think this percentage will increase? Yes/No

- Why?...

\section{References}

1. Ellis H, Moran BJ, Thompson JN et al (1999) Adhesion-related hospital readmissions after abdominal and pelvic surgery: a retrospective cohort study. Lancet 353:1476-1480

2. Parker MC, Ellis H, Moran BJ et al (2001) Postoperative adhesions: ten-year follow-up of 12, 584 patients undergoing lower abdominal surgery. Dis Colon Rectum 44:822-829 discussion 829-830

3. Sulaiman H, Gabella G, Davis MC et al (2001) Presence and distribution of sensory nerve fibers in human peritoneal adhesions. Ann Surg 234:256-261

4. Vrijland WW, Jeekel J, van Geldorp HJ et al (2003) Abdominal adhesions: intestinal obstruction, pain, and infertility. Surg Endosc 17:1017-1022

5. Van Der Krabben AA, Dijkstra FR, Nieuwenhuijzen $M$ et al (2000) Morbidity and mortality of inadvertent enterotomy during adhesiotomy. Br J Surg 87:467-471

6. Wullstein C, Gross E (2003) Laparoscopic compared with conventional treatment of acute adhesive small bowel obstruction. Br J Surg 90:1147-1151

7. Szomstein S, Lo Menzo E, Simpfendorfer C et al (2006) Laparoscopic lysis of adhesions. World J Surg 30:535-540

8. Wiseman DM, Trout JR, Diamond MP (1998) The rates of adhesion development and the effects of crystalloid solutions on adhesion development in pelvic surgery. Fertil Steril 70:702-711

9. van den Tol MP, Haverlag R, van Rossen ME et al (2001) Glove powder promotes adhesion formation and facilitates tumour cell adhesion and growth. Br J Surg 88:1258-1263

10. Kumar S, Wong PF, Leaper DJ (2009) Intra-peritoneal prophylactic agents for preventing adhesions and adhesive intestinal obstruction after non-gynaecological abdominal surgery. Cochrane Database Syst Rev 1:CD005080

11. Wiseman DM, Trout JR, Franklin RR et al (1999) Meta-analysis of the safety and efficacy of an adhesion barrier (Interceed TC7) in laparotomy. J Reprod Med 44:325-331

12. Brown CB, Luciano AA, Martin D et al (2007) Adept (icodextrin $4 \%$ solution) reduces adhesions after laparoscopic surgery for 
adhesiolysis: a double-blind, randomized, controlled study. Fertil Steril 88:1413-1426

13. Rajab TK, Wallwiener M, Talukdar S et al (2009) Adhesionrelated complications are common, but rarely discussed in preoperative consent: a multicenter study. World J Surg 33:748-750

14. Trew G, Cooke I, Lower A et al (2009) Post-operative abdominal adhesions-awareness of UK gynaecologists-a survey of members of the Royal College of Obstetricians and Gynaecologists. J Gynecol Surg 6:25-37

15. Ellis H, Crowe A (2009) Medico-legal consequences of postoperative intra-abdominal adhesions. Int J Surg 7:187-191

16. Miller G, Boman J, Shrier I et al (2000) Etiology of small bowel obstruction. Am J Surg 180:33-36

17. Miller G, Boman J, Shrier I et al (2000) Natural history of patients with adhesive small bowel obstruction. Br J Surg 87: 1240-1247

18. Bizer LS, Liebling RW, Delany HM et al (1981) Small bowel obstruction: the role of nonoperative treatment in simple intestinal obstruction and predictive criteria for strangulation obstruction. Surgery 89:407-413

19. Edna TH, Bjerkeset T (1998) Small bowel obstruction in patients previously operated on for colorectal cancer. Eur J Surg 164: 587-592

20. Beck DE, Opelka FG, Bailey HR et al (1999) Incidence of smallbowel obstruction and adhesiolysis after open colorectal and general surgery. Dis Colon Rectum 42:241-248

21. Parker MC, Wilson MS, Menzies D et al (2005) The SCAR-3 study: 5-year adhesion-related readmission risk following lower abdominal surgical procedures. Colorectal Dis 7:551-558

22. Stanciu D, Menzies D (2007) The magnitude of adhesion-related problems. Colorectal Dis 9(Suppl 2):35-38

23. Wilson MS (2007) Practicalities and costs of adhesions. Colorectal Dis 9(Suppl 2):60-65

24. Lower AM, Hawthorn RJ, Clark D et al (2004) Adhesion-related readmissions following gynaecological laparoscopy or laparotomy in Scotland: an epidemiological study of 24046 patients. Hum Reprod 19:1877-1885
25. Hackethal A, Sick C, Brueggmann D et al (2010) Awareness and perception of intra-abdominal adhesions and related consequences: survey of gynaecologists in German hospitals. Eur J Obstet Gynecol Reprod Biol 150(2):180-189

26. Beck DE, Cohen Z, Fleshman JW et al (2003) A prospective, randomized, multicenter, controlled study of the safety of Seprafilm adhesion barrier in abdominopelvic surgery of the intestine. Dis Colon Rectum 46:1310-1319

27. Menzies D, Pascual MH, Walz MK et al (2006) Use of icodextrin $4 \%$ solution in the prevention of adhesion formation following general surgery: from the multicentre ARIEL Registry. Ann R Coll Surg Engl 88:375-382

28. Parker MC, Wilson MS, van Goor H et al (2007) Adhesions and colorectal surgery-call for action. Colorectal Dis 9(Suppl 2): $66-72$

29. Cabana MD, Rand CS, Powe NR et al (1999) Why don't physicians follow clinical practice guidelines? A framework for improvement. JAMA 282:1458-1465

30. Kosaka H, Yoshimoto T, Yoshimoto T et al (2008) Interferongamma is a therapeutic target molecule for prevention of postoperative adhesion formation. Nat Med 14:437-441

31. Chokshi NK, Simeone DM, Chari RS et al (2009) A survey of academic surgeons: work, stress, and research. Surgery 146: 462-468

32. Dirmaier J, Harfst T, Koch U et al (2007) Incentives increased return rates but did not influence partial nonresponse or treatment outcome in a randomized trial. J Clin Epidemiol 60:1263-1270

33. Edwards PJ, Roberts I, Clarke MJ et al (2009) Methods to increase response to postal and electronic questionnaires. Cochrane Database Syst Rev 3:MR000008

34. Jeekel H (1997) Cost implications of adhesions as highlighted in a European study. Eur J Surg 163(Suppl 579):43-45

35. Tingstedt B, Isaksson J, Andersson R (2007) Long-term followup and cost analysis following surgery for small bowel obstruction caused by intra-abdominal adhesions. Br J Surg 94:743-748 